\title{
Piracy prevention and the pricing of information goods*
}

\author{
Helmuth Cremer ${ }^{\dagger}$ and Pierre Pestieau ${ }^{\ddagger}$ \\ November 2005, this version: February 2006 \\ CORE Discussion Paper 2006/18
}

\begin{abstract}
This paper develops a simple model of piracy to analyze its effects on prices and welfare and to study the optimal enforcement policy. A monopolist produces an information good (involving a "large" development cost and a "small" reproduction cost) that is sold to two groups of consumers differing in their valuation of the good. We distinguish two settings: one in which the monopoly is regulated and one in which it maximizes profits and is not regulated, except that the public authority may be responsible for the control of piracy. We show that copying or piracy might be welfare enhancing because it is a way to "provide" the good to some individuals (those with a low willingness to pay) without undermining the firm's ability to finance the development cost via the pricing scheme applied to high valuation consumers. The level of piracy control differs according to the regulatory environment. Three levels of piracy control emerge. The highest is the one chosen by the private monopoly. The next level is the one chosen by the regulated monopoly. The lowest, that can be zero, is the level of control chosen by the public authority when the good is sold (and priced) by a private monopoly.
\end{abstract}

JEL classification: L11, L86, D82

Keywords: piracy, copying, intellectual property, information good.

\footnotetext{
${ }^{*}$ We thank Philippe De Donder for his comments.

${ }^{\dagger}$ IDEI, University of Toulouse.

${ }^{\ddagger}$ CREPP, University of Liège, CORE, Delta and CEPR.
} 


\section{Introduction}

Over the last years, the economics of information goods (e.g. DVD, CD, software) has attracted the attention of economists, both empirical and theoretical. One of the key characteristics of information goods is that they can be copied with little, if any, quality degradation and quite often at a rather low cost. Illegal copying and piracy are now a big issue. They threaten the financial sustainability of the whole industry.

At a theoretical level, the question of prevention and control is central. Nobody disputes that some copy protection is needed. The question is how much. ${ }^{1}$

There exists now an impressive literature on the issue of piracy and copying and more specifically on the question of piracy prevention and copyrights protection. For a survey, see, e.g., Peitz and Waelbroeck (2004). In particular, a number of papers have shown that some degree of piracy may increase the sellers profits either because of "indirect appropriability" ${ }^{2}$ or because of network effects ${ }^{3}$

We are interested by the welfare implication of copying and piracy. The cost of these practices on the industry is quite obvious; the benefits for some consumers are also quite clear. We consider a monopolist who produces an information good (involving a "large" development cost and a "small" reproduction cost) that is sold to two groups of consumers differing in their valuation of the good. The consumers with a low willingness to pay can copy the information good at some cost and some quality loss. The consumers with a high willingness to pay cannot engage in fully fledged piracy. However, they can consume the version designed for the other customer type. We show that some copying or piracy might be desirable for efficiency reasons. In our setting piracy may be an effective way to "provide" the good to some individuals (those with a low willingness to pay) without undermining the ability of the provider to finance the development cost of the information good through the pricing scheme applied to high willingness to pay consumers.

Our paper is close to that of Takeyama (1994) who also studies a monopolist selling

\footnotetext{
${ }^{1}$ See Romer (2002).

${ }^{2}$ See Liebowitz (1985). The idea is that sellers may be able to indirectly extract the surplus created from copying by charging a higher price to the legal buyer.

${ }^{3}$ See e.g., Connert and Rumelt (1991), Takeyama (1994) and Shy and Thisse (1999).
} 
reproducible good to two groups of consumers differing in their valuation of the good. Because of network externalities this valuation increases with the number of users. The monopolist cannot price discriminate. Consequently, in the absence of copying it will either serve all consumers at a relatively low price or only the high valuation consumers at a price that makes their participation constraint binding. When copying is introduced, a new equilibrium emerges with high valuation consumers buying the original and the low valuation consumer buying the copy. Such an equilibrium may yield a higher profit than that the producer can obtain in the absence of piracy. This is because the consumption of the individuals who consume an illegal copy increases the willingness to pay of the consumers who buy from the producer. It is also superior in terms of welfare.

Our paper does not assume network externalities but allows for price segmentation. Thus in our setting piracy is not a device to overcome the constraints imposed by linear pricing. Nevertheless, it enhances the monopolists opportunities to differentiate prices between customers. Another distinctive feature of our paper is that it is concerned with the desirability of piracy control. ${ }^{4}$ We distinguish different settings: one in which the monopoly is fully regulated by a public authority and one in which the monopoly is free except that the public authority may be responsible for the control of piracy. We end up with three levels of piracy control. The highest is the one chosen by the private monopoly when there is no interference from a regulator. The next level is the one chosen by the regulated monopoly. The lowest, that can be zero, is the level of control chosen by the public authority in case of private monopoly.

\section{The model}

We consider a simple economy with two types of consumers indexed by $i=1,2$. The number of type 1 individuals is normalized to 1 , while that of type 2 individuals is denoted by $n$. There are two goods: an information good $Y$ and a numeraire $X$. One can think about the two types of individuals for instance as "professional" versus "household" customers for software or "institutional" versus "individual" subscribers

\footnotetext{
${ }^{4}$ The determination of the degree of copyright protection has also been studied by Yoon (2002).
} 
in the case of scientific journals. Each individual consumes at most one unit of the information good of quality $y_{i} \geq 0(i=1,2)$. By definition $y_{i}=0$ means that type $i$ individuals do not consume the information good. Individual are endowed with $m_{i}$ units of the numeraire; their consumption of the numeraire good is denoted by $x_{i}$.

The information good is supplied by a single producer at a price $P$ which is a nonlinear function of its quality. Without loss of generality we assume that the producer provides two bundles $(Y, P)$, namely $\left(y^{H}, p^{H}\right)$ and $\left(y^{L}, p^{L}\right)$, with $y^{H} \geq y^{L}$. In other words, two variants of the information goods are available. A high quality variant designed for professional customers (type 1) and a low quality variant designed for households (type 2 ). In addition all individuals can consume the bundle $(0,0)$ i.e., they have the option not to consume the information good at all. Observe that $y^{H}=y^{L}$ is not ruled out; in the case we continue to think about $y^{H}$ as the variant designed for professional customers which may be priced differently from $y^{L} .{ }^{5}$ For instance institutional and individual rates for journal subscription differ, while the product is essentially the same.

The cost (expressed in units of the numeraire) of producing respectively $n^{H}$ and $n^{L}$ units of the two variants of the information good $y^{H}$ and $y^{L}$ (with $y^{H} \geq y^{L}$ ) is given by

$$
C=F\left(y^{H}\right)+n^{H} c\left(y^{H}\right)+n^{L} c\left(y^{L}\right) .
$$

This cost function, inspired by Cremer and Laffont (2003) reflects the specific nature of the information good. One can think of $F$ as the development cost (that is determined by the highest available quality) while $c$ is the "reproduction" cost. When $c$ is zero, $Y$ is a pure non-rival good. On the other hand, when $F=0$, we have a private good (with constant return to scale production technology).

The preferences of the first group of consumers, are represented by the following expression:

$$
U_{1}(Y, P)=\left\{\begin{array}{lll}
u_{1}\left(y^{H}\right)+m_{1}-p^{H} & \text { if } & (Y, P)=\left(y^{H}, p^{H}\right), \\
u_{1}\left(y^{L}\right)+m_{1}-p^{L}-K & \text { if } & (Y, P)=\left(y^{L}, p^{L}\right), \\
m_{1} & \text { if } & (Y, P)=(0,0),
\end{array}\right.
$$

\footnotetext{
${ }^{5}$ This may be possible because of the specific features of the type's preferences for the information good; see below.
} 
where $u_{1}$ is increasing and concave $\left(u_{1}^{\prime}>0, u_{1}^{\prime \prime}<0\right)$ with $u_{1}(0)=0$. These preferences have the specific feature that type 1 consumers incur a utility loss of $K \geq 0$ when they consume the variant designed for the other type irrespective of the effective quality differential. We assume that group 1 cannot engage in "fully-fledged piracy". For instance, a library in a research center can cheat (at a reputation cost of $K$ ) by using an individual subscription to a journal; however, we assume that it cannot put a photocopied issue on its shelves.

The second group consists of $n$ individuals who can pirate the information good at some cost $k$ and some (proportional) utility loss of $(1-\theta)$. Their utility is given by:

$$
U_{2}(Y, P)=\left\{\begin{array}{lll}
u_{2}\left(y^{L}\right)+m_{2}-p^{L} & \text { if } \quad(Y, P)=\left(y^{L}, p^{L}\right) \\
\theta u_{2}\left(y^{L P}\right)+m_{2}-k\left(y^{L P}, e\right) & \text { if } \quad(Y, P)=(0,0)
\end{array}\right.
$$

where $u_{2}$ is increasing and concave $\left(u_{2}^{\prime}>0, u_{2}^{\prime \prime}<0\right)$ with $u_{2}(0)=0$. Further we have $\theta \leqslant 1 ; y^{L P} \geq 0$ is the quality of the illegal copy, while $k=k\left(y^{L P}, e\right)$ is its cost function which depends on $e$, the monetary effort to enforce copyrights (to prevent piracy). This function satisfies $k(0, e)=0$; using subscripts to denote its partial derivatives we have $k_{y}>0, k_{y y}>0$ and (as long as $\left.y^{L P}>0\right) k_{e}>0$ and $k_{e e}<0 .{ }^{6}$ For consistency $y^{L P}=0$ represents the case where there is no piracy, in which case $U_{2}(0,0)=m_{2}$.

For a given enforcement effort, the quality of the copy is the solution to: ${ }^{7}$

$$
y^{L P}=\arg \max _{y}\left[\theta u_{2}(y)-k(y, e)\right]
$$

subject to $y \leq y^{H}$. Since individuals have the option of not engaging in piracy we necessarily have $\theta u_{2}\left(y^{L P}\right)-k\left(y^{L P}, e\right) \geq 0$. It is assumed that the individual's copying technology is never more efficient that the firm's technology; i.e., for any $y$ and $e$ we have

$$
k(y, 0) \geqslant c(y),
$$

\footnotetext{
${ }^{6}$ The assumption on $u_{i}$ and $k$ are stronger than necessary.

${ }^{7}$ The second order condition

$$
\theta u_{2}^{\prime \prime}(y)-k_{y y}(y, e)<0
$$
}

is satisfied per our assumptions on $u_{2}$ and $k$. 
and

$$
k_{y}(y, e) \geqslant c^{\prime}(y)
$$

Finally, to ensure single crossing we assume to satisfy $u_{1}(y)>u_{2}(y)$ (for any $y>0$ ) and $u_{1}^{\prime}(y)>u_{2}^{\prime}(y)$ (for any $\left.y \geq 0\right)$.

We shall now characterize the optimal allocation under various assumptions regarding the scope of regulation and the information structure. In the first part, we assume that the provider of the information good is regulated and that the same regulatory authority also controls $e$, the copyright enforcement effort. Three information structures are studied. First, we consider the case of full information. Then we assume that individual types continue to be observable but we allow for the possibility of piracy. Finally, it is assumed that types are not observable (while piracy continues to be possible). The last setting is obviously the most interesting one, but the first two settings provide interesting benchmarks. In the second part of the paper, we assume that the provider of the information good is an unregulated (profit-maximizing) monopolist. This monopolist may or may not be in charge of copyright enforcement. In particular, we consider the case where the enforcement policy is entrusted to a welfare maximizing authority (while the pricing of the information good is set by the monopoly).

\section{$3 \quad$ Regulated provider}

Assume that the objective of the regulatory authority is given by

$$
W=U_{1}+n U_{2}+(1+\lambda) \pi
$$

where $\lambda \geq 0$ is the cost of public funds while $\pi$ is the (negative or positive) profit of the supplier of the information good. Recall that consumption of the information good is voluntary; all individuals have the option of not consuming the good. Consequently, the individual participation constraints apply in all the settings considered.

\subsection{Full information optimum}

Let us assume for the time being that individual types are observable and that there is no possibility of piracy. The so defined full information solution is obtained by maximizing 
(6) with respect to $\left(y^{H}, p^{H}\right),\left(y^{L}, p^{L}\right)$ and $e$, subject to the participation constraints $U_{1} \geq m_{1}$ and $U_{2} \geq m_{2}$. The provider's profit $\pi$ is defined by

$$
\pi=p^{H}+n p^{L}-F\left(y^{H}\right)-c\left(y^{H}\right)-n c\left(y^{L}\right)-e .
$$

For simplicity profits are net of enforcement cost $e$. Alternatively they could have been deducted directly in (6); with a regulated firm this would be completely equivalent.

It is plain that both participation constraints are binding at the full information optimum. ${ }^{8}$ Consequently, we have $U_{1}=m_{1}$ and $U_{2}=m_{2}$, which from (1) and (2) implies $p^{H}=u_{1}\left(y^{H}\right)$ and $p^{L}=u_{2}\left(y^{L}\right)$. Substituting these expression into (6) while making use of (6) yields the following expression for welfare ${ }^{9}$

$$
W^{F I}=(1+\lambda)\left[u_{1}\left(y^{H}\right)+n u_{2}\left(y^{L}\right)-F\left(y^{H}\right)-c\left(y^{H}\right)-n c\left(y^{H}\right)-e\right] .
$$

The maximization of $W^{F I}$ yields of course $e=0$; under full information, enforcement expenditures have no benefits and are set at zero. As for the level of $y$, two regimes can arise depending on whether $y^{H}>y^{L}$ or $y^{H}=y^{L}$ :

- Regime $A: y^{H}>y^{L}$, with

$$
\begin{aligned}
& u_{1}^{\prime}\left(y^{H}\right)=F^{\prime}\left(y^{H}\right)+c^{\prime}\left(y^{H}\right) ; \\
& u_{2}^{\prime}\left(y^{L}\right)=c^{\prime}\left(y^{L}\right) .
\end{aligned}
$$

- Regime $B: y^{H}=y^{L}=y$, with

$$
u_{1}^{\prime}(y)+n u_{2}^{\prime}(y)=F^{\prime}(y)+c^{\prime}(y)+n c^{\prime}(y)
$$

To understand these conditions, let us start with Regime $B$, where all individuals consume the same level of the information good. The optimal provision is then characterized by (11) which is effectively the Samuelson condition: the optimal quality is that

\footnotetext{
${ }^{8}$ This is necessarily true when $\lambda>0$. When $\lambda=0$, the solution is not unique, but one of the solutions is such that both participation constraints are binding; see below for further discussion of the case where $\lambda=0$.

${ }^{9}$ In expression (8) and in all subsequent expresion for welfare we have dropped the (constant) terms $m_{1}$ and $m_{2}$ for notational convenience. Strictly speaking $W$ is thus "surplus" or "rent" (utility in excess of the reservation level).
} 
for which the aggregate willingness to pay is equal to the aggregate marginal cost. This type of solution necessarily occurs when the reproduction cost is zero (nonrival good). With monotonic preferences it is then never optimal to have one group consume less than the full quality available. When reproduction costs are sufficiently high (and/or the willingness to pay of type 2 customers sufficiently small) we can have Regime $A$, where only the individuals with a high willingness to pay consume the full quality variant. The Samuelson condition then only accounts for type 1 individuals while $y^{L}$ is determined so that the marginal reproduction cost equals the marginal willingness to pay. ${ }^{10}$

Observe that the level of $\lambda$ is of no relevance for this solution. The levels of $Y$ are effectively the Pareto-efficient ones. This is because (under full information) the entire consumer surplus is extracted through nonlinear pricing. Consequently, the problem is equivalent to the maximization of total surplus which, with quasi-linear preferences, yields the Pareto-efficient levels of $Y^{11}$

\subsection{Full information except for piracy}

Let us now assume that types continue to be observable, while introducing the possibility of piracy for type 2 consumers. In other words the seller of the information good can identify type 1 but he cannot (costlessly) prevent type 2 from engaging in piracy. This changes the problem only to the extent that the participation constraint of type 2 now requires

$$
u_{2}\left(y^{L}\right)-p^{L} \geq \theta u_{2}\left(y^{L P}\right)-k\left(y^{L P}, e\right) \geq 0 .
$$

In other words, individuals of type 2 must have at least the utility level they could obtain by consuming the pirated version of the information good. Observe that in this setting, it will never be optimal to let consumers effectively engage in piracy. This is because the copying technology is less efficient than the firm's technology; see assumption (4).

\footnotetext{
${ }^{10}$ This is exactly similar to a public good problem when saturation is possible; see also Cremer and Laffont (2003).

${ }^{11}$ With quasi-linear preferences, the levels of $Y$ are the same at all Pareto-efficient allocations.
} 
Substituting the participation constraint into (6), making use of (7) and rearranging yields the following expression for welfare

$$
\begin{aligned}
W^{F I P}= & (1+\lambda)\left[u_{1}\left(y^{H}\right)+n u_{2}\left(y^{L}\right)-F\left(y^{H}\right)-c\left(y^{H}\right)-n c\left(y^{L}\right)-e\right] \\
& -n \lambda\left[\theta u_{2}\left(y^{L P}\right)-k\left(y^{L P}, e\right)\right]
\end{aligned}
$$

where $y^{L P}$ is determined according to (3). The first term on the RHS of this expression corresponds to $W^{F I}$ as defined by (8) and the second term measures the social cost of the extra rent that has to be conceded to type 2 customers because the possibility of piracy increases their reservation utility level. This second term does not depend on $y^{H}$ and $y^{L}$. Consequently we obtain the same levels of $y^{H}$ and $y^{L}$ as in the previous subsection (i.e., expression (9)-(11). The new feature brought about by the possibility of piracy is that the level of $e$ is now determined by

$$
k_{e}\left(y^{L P}, e\right)=\frac{1+\lambda}{n \lambda} .
$$

Not surprisingly, the optimal level of enforcement is thus determined to equalize marginal cost, $(1+\lambda)$, to marginal benefits $n \lambda k_{e}\left(y^{L P}, e\right)$. Benefits arise because the reservation utility of type 2 individuals decreases by $k_{e}\left(y^{L P}, e\right)$ and the revenue extracted increases by $n k_{e}\left(y^{L P}, e\right)$. The induced increase in welfare is equal to this extra revenue multiplied by the cost of public funds $\lambda$.

\subsection{Asymmetric information}

We now turn to a fully fledged setting of asymmetric information by assuming that a consumer's type is not publicly observable. In addition, piracy remains possible for type 2 individuals. When individual types are not publicly observable, the consumption bundles $\left(y^{H}, p^{H}\right)$ and $\left(y^{L}, p^{L}\right)$ must be incentive compatible (so that no type prefers the other type's consumption bundle). In our setting, it is plain that the relevant incentive constraint will be that of type 1 which is given by

$$
u_{1}\left(y^{H}\right)-p^{H} \geq u_{1}\left(y^{L}\right)+p^{L}-K .
$$

It is easy to show that when $K=0$, this constraint will necessarily be binding. When $K$ is large enough, on the other hand, (15) may not be binding. In other words, it 
is not impossible that the solution obtained in subsection (3.2) satisfies (15) and thus continues to be feasible when types are not observable. To reduce the number of cases to be considered we shall assume throughout the remainder of the paper that (15) is binding.

When types were observable it was always optimal to "prevent" piracy by setting the price of the legal product sufficiently low (i.e., to satisfy 12). This is because per our assumptions on cost and preferences, it is optimal to provide the information good to all individuals and the legal technology is more efficient. With asymmetric information, however, it may be optimal for the provider to exclude consumers with a low willingness to pay, so that a higher price can be charged to the consumers of type 1 . In that case, we may have a solution where individuals of type 2 consume the pirated good.

We shall consider the two cases separately.

\section{Case I: No piracy: $Y$ is sold to both groups}

Assume, for the time being, that the consumption bundles $\left(y^{H}, p^{H}\right)$ and $\left(y^{L}, p^{L}\right)$ are such that (12) is satisfied. In other words, individuals of type 2 prefer to buy $\left(y^{L}, p^{L}\right)$ rather then consume the pirated version. The Lagrangean expression of the regulatory authority's problem is then given by:

$$
\begin{aligned}
\mathcal{L}_{I}= & u_{1}\left(y^{H}\right)-p^{H}+n\left[u_{2}\left(y^{L}\right)-p^{L}\right] \\
& +(1+\lambda)\left[p^{H}+n p^{L}-F\left(y^{H}\right)-c\left(y^{H}\right)-n c\left(y^{L}\right)-e\right] \\
& +\eta_{1}\left[u_{1}\left(y^{H}\right)-p^{H}\right] \\
& +\eta_{2}\left[u_{2}\left(y^{L}\right)-p^{L}-\theta u_{2}\left(y^{L P}\right)+k\left(y^{L P}, e\right)\right] \\
& +\mu\left[u_{1}\left(y^{H}\right)-p^{H}-u_{1}\left(y^{L}\right)+p^{L}+K\right] .
\end{aligned}
$$

where $\lambda \geq 0$ is the cost of public funds, $\eta_{i}$ is the multiplier of the participation constraint of type $i=1,2$, while $\mu$ is the multiplier associated with the self-selection constraint (of 
type 1). ${ }^{12}$ The first-order conditions are given by

$$
\begin{aligned}
& \frac{\partial \mathcal{L}_{I}}{\partial y^{H}}=u_{1}^{\prime}\left(y^{H}\right)-(1+\lambda)\left[F^{\prime}\left(y^{H}\right)+c^{\prime}\left(y^{H}\right)\right]+\left(\eta_{1}+\mu\right) u_{1}^{\prime}\left(y^{H}\right)=0 \\
& \frac{\partial \mathcal{L}_{I}}{\partial y^{L}}=n u_{2}^{\prime}\left(y^{L}\right)-(1+\lambda) n c^{\prime}\left(y^{L}\right)+\eta_{2} u_{2}^{\prime}\left(y^{L}\right)-\mu u_{1}^{\prime}\left(y^{L}\right)=0 \\
& \frac{\partial \mathcal{L}_{I}}{\partial p^{H}}=\lambda-\left(\eta_{1}+\mu\right)=0 \\
& \frac{\partial \mathcal{L}_{I}}{\partial p^{L}}=n \lambda-\eta_{2}+\mu=0 \\
& \frac{\partial \mathcal{L}_{I}}{\partial e}=-(1+\lambda)+\eta_{2} k_{e}\left(y^{L P}, e\right)=0
\end{aligned}
$$

As mentioned above, we shall concentrate on the case where $K$ is not too large so that $\mu>0$ and $\eta_{1}=0$ (maximum price that can be charged to type 1 individuals is determined by the incentive constraint rather then by the participation constraint). ${ }^{13}$ Like in the full information optimum we have to distinguish between two regimes depending on whether $y^{H}>y^{L}$ or $y^{H}=y^{L}$.

Regime $A: y^{H}>y^{L}$. Combining (16) and (18) yields

$$
u_{1}^{\prime}\left(y^{H}\right)=F^{\prime}\left(y^{H}\right)+c^{\prime}\left(y^{H}\right)
$$

which is the full-information condition (9). This is the usual no distortion at the top result. Consequently the level supplied to type 1 customers is the same as in the full information solution. However, the price is lower since we now have $p^{H}<u_{1}\left(y^{H}\right)$; because of the incentive constraint type 1 consumers have some informational rent. ${ }^{14}$ Turning to type 2 , when $\eta_{1}=0$ equation (18) implies $\mu=\lambda$ and we have from (17) and (19):

$$
u_{2}^{\prime}\left(y^{L}\right)=c^{\prime}\left(y^{L}\right)+\frac{\lambda}{n(1+\lambda)}\left[u_{1}^{\prime}\left(y^{L}\right)-u_{2}^{\prime}\left(y^{L}\right)\right]
$$

\footnotetext{
${ }^{12}$ We ignore the incentive constraint of type 2, which is never binding.

${ }^{13}$ When $\mu=0$ and $\eta_{1}>0$, we have the same results as in Subsection 3.2.

${ }^{14}$ Combining the incentive constraint and the participation constraint of type 2 individuals we have

$$
\begin{aligned}
u_{1}\left(y^{H}\right)-p^{H}-K= & u_{1}\left(y^{L}\right)+p^{L}> \\
& u_{2}\left(y^{L}\right)-p^{L} \geq 0 .
\end{aligned}
$$
}


where $u_{1}^{\prime}\left(y^{L}\right)-u_{2}^{\prime}\left(y^{L}\right)>0$. Consequently, the quality of the information good sold to type 2 individuals is lower than under full information. This result does not come as a surprise; it is a standard property in the nonlinear pricing literature. The underlying intuition is that reducing the quality supplied to type 2 , relaxes the incentive constraint, so that more money can be extracted from type 1 individuals.

Expenditures on enforcement, $e$, are characterized by (20) which with $\mu=\lambda$ yields

$$
k_{e}\left(y^{L P}, e\right)=\frac{1+\lambda}{n \lambda+\lambda}=\frac{1+\lambda}{(n+1) \lambda}
$$

Comparing with (14) shows that the presence of asymmetric information increases the benefits of enforcement expenditures. These benefits are now given by $(n+1) \lambda k_{e}\left(y^{L P}, e\right)$. When types are not observable an increase in the cost of piracy yields a double benefit. It decreases the reservation utility of type 2 consumers exactly like in the case where types could be observed (Subsection 3.2). In addition, it now also reduces the informational rents of type 1 individuals (by way of the incentive constraint). This argument in itself is, however, not sufficient to compare the levels of $e$ implied by (14) and (22). Such a comparison depends on the level of $y^{L P}$ which is endogenous and will not be the same in the two settings (except in special cases). To be more precise $e$ and $y^{L P}$ are jointly determined by the following system of equations

$$
\begin{aligned}
& k_{e}\left(y^{L P}, e\right)=\Lambda, \\
& \theta u_{2}^{\prime}\left(y^{L P}\right)-k_{y}\left(y^{L P}, e\right)=0,
\end{aligned}
$$

where $\Lambda$ is the RHS of (14) or (22), while (24) is the first-order condition associated with (3). Differentiating (23)-(24) and rearranging yields

$$
\frac{d e}{d \Lambda}=\frac{\left[\theta u_{2}^{\prime \prime}-k_{y y}\right]}{k_{e y}^{2}+k_{e e}\left(\theta u_{2}^{\prime \prime}-k_{y y}\right)}<0 .
$$

Since the RHS of $(22)$, namely $(1+\lambda) /(n+1) \lambda$, is smaller than that of (14), namely $(1+\lambda) / n \lambda$, it thus follows that the asymmetry of information about individual types leads to a higher level of copyright enforcement. Observe that this result is conditional on the assumption that type 2 individuals are served at the second-best solution (i.e., Case $I$ is relevant). 
Like under full information, Regime $A$ occurs when reproduction $\operatorname{costs} c$ are relatively high; when $c$ is sufficiently small, we are close to the case of a non-rival good and it will be optimal to offer the same quality to the two types and we have Regime $B$.

Regime $B: y^{F}=y^{L}=y$. Observe that this regime does not necessarily imply that the two types pay the same price. Because type 1 incurs a reputation cost when mimicking type 2 , they can be charged a higher price for their variant of the product, even when this is not of higher quality than that sold to the other type. This possibility is illustrated for instance by the fact that library subscription rates to journals can be much higher than individual rates even though the product is essentially the same.

The first-order condition with respect to $y^{F}=y^{L}=y$ is simply given by the sum of (16) and (17). The other first-order condition remain unchanged. Concentrating once again on the case where $\eta_{1}=0$ (so that $\mu=\lambda$ ) we obtain the following optimality condition for $y$ :

$$
u_{1}^{\prime}(y)+n u_{2}^{\prime}(y)=F^{\prime}(y)+c^{\prime}(y)+n c^{\prime}(y)+\frac{\lambda}{(1+\lambda)}\left[u_{1}^{\prime}(y)-u_{2}^{\prime}(y)\right] .
$$

Comparing this condition to its full-information counterpart, expression (11) shows that under Regime B the presence of asymmetric information leads to a lower quality being supplied to both types of individuals. This downward distortion arises from the incentive term $\lambda /(1+\lambda)\left[u_{1}^{\prime}(y)-u_{2}^{\prime}(y)\right]{ }^{15}$ Lowering the quality from the full-information level does have a larger impact on the mimicking individual than on the mimicked one. Consequently, it relaxes an otherwise binding incentive constraint so that more revenue can be extracted from the type 1 consumers.

Finally note that enforcement expenditures are the same as under Regime A; they continue to be determined by $(22) .{ }^{16}$

\subsubsection{Case $I I$ : Piracy: $Y$ is sold to group 1 only}

As an alternative to the policy considered in Case 1, there is the possibility of not serving group 2 at all. Then, type 1 customers no longer have the possibility of mimicking type

\footnotetext{
${ }^{15}$ Recall that $\mu=\lambda$.

${ }^{16}$ Consequently, $y^{L P}$ as determined by $(24)$ is the same as in the other regime although $y^{L}$ is not.
} 
2. To be more precise, mimicking type 2 is equivalent to not consuming the good at all. Recall that, by assumption, type 1 individuals do not have the possibility to engage in piracy per se. Consequently, the seller will be able to extract the entire surplus from type 1 individuals. Individuals of type 2, on the other hand, can consume a pirated version of the good and their utility level is given by $\theta u_{2}\left(y_{L}^{p}\right)-k\left(y_{L}^{p}, e\right)$, where $y^{L P}$ is determined by (3). The variables which are left to be determined are $y^{H}, p^{H}$ and $e$ and the Lagrangean expression of the regulator's problem is given by

$$
\begin{aligned}
\mathcal{L}_{I I}= & u_{1}\left(y^{H}\right)-p^{H}+n\left[\theta u_{2}\left(y^{L P}\right)-k\left(y^{L P}, e\right)\right] \\
& +(1+\lambda)\left[p^{H}-F\left(y^{H}\right)-c\left(y^{H}\right)-e\right] \\
& +\eta_{1}\left[u_{1}\left(y^{H}\right)-p^{H}\right] .
\end{aligned}
$$

The solution to this problem yields the full information level of $y^{H}$, specified by (9). This is exactly the same solution as in Case $I$. However, the level of $p^{H}$ is different for we return to the full information solution with $p^{H}=u_{1}\left(y^{H}\right)$. Furthermore we have

$$
\frac{\partial \mathcal{L}_{I I}}{\partial e}=-n k\left(y^{L P}, e\right)-(1+\lambda)
$$

so that $e=0$. Expenditures on enforcement are not only costly (second term), but their "benefits" are negative. Piracy has no impact on the revenue of the firm; consequently, it is optimal to let individuals of type 2 consume the pirated version. This is a way to "provide" the good to this class of customers without interfering with the incentive constraint (and thus with the revenue that can be extracted from type 1 customers). In other words, when Case II occurs the possibility of piracy is effectively welfare enhancing. This is also reflected in the pattern of enforcement expenditures. In Case $I$, these expenditures were higher than when types were observable (Subsection 3.2). Here, they are lower and effectively drop to zero.

\section{Profit maximizing monopoly}

So far we have assumed that the provider of the information good was a regulated firm so that its pricing policy was set to maximize welfare. We now consider a different setting wherein the firm is a profit maximizing monopoly. We first study its pricing policy (the 
bundles $\left(y^{H}, p^{H}\right)$ and $\left.\left(y^{L}, p^{L}\right)\right)$ for a given level of $e$. Then we study the determination of the level of enforcement assuming that it is either chosen by the monopoly or, more interestingly, by a welfare maximizing authority.

\subsection{Pricing of $Y$}

Let us concentrate for the time being on Case $I$. The Lagrangean expression associated with the monopoly's problem is given by:

$$
\begin{aligned}
\mathcal{L}_{M I}= & {\left[p^{H}+n p^{L}-F\left(y^{H}\right)-c\left(y^{H}\right)-n c\left(y^{L}\right)-e\right] } \\
& +\eta_{1}\left[u_{1}\left(y^{H}\right)-p^{H}\right] \\
& +\eta_{2}\left[u_{2}\left(y^{L}\right)-p^{L}-\theta u_{2}\left(y^{L P}\right)+k\left(y^{L P}, e\right)\right] \\
& +\mu\left[u_{1}\left(y^{H}\right)-p^{H}-u_{1}\left(y^{L}\right)+p^{L}+K\right] .
\end{aligned}
$$

The first-order conditions are the following

$$
\begin{aligned}
& -\left[F^{\prime}\left(y_{H}\right)+c^{\prime}\left(y_{H}\right)\right]+\left(\eta_{1}+\mu\right) u_{1}^{\prime}\left(y_{H}\right)=0, \\
& -n c^{\prime}\left(y_{L}\right)+\eta_{2} u_{2}^{\prime}\left(y_{L}\right)-\mu u_{1}^{\prime}\left(y_{L}\right)=0 \\
& 1-\left(\eta_{1}+\mu\right)=0 \\
& n-\eta_{2}+\mu=0 .
\end{aligned}
$$

Assuming once again that $\eta_{1}=0$ and $\mu>0$, one easily shows that under Regime $A$ (i.e., when $\left.y^{H}>y^{L}\right), y^{H}$ continues to be determined by the full information condition (9); not surprisingly, the no distortion at the top property continues to apply. Furthermore, (28) and (29) imply

$$
u_{2}^{\prime}\left(y^{L}\right)=c^{\prime}\left(y^{L}\right)+\frac{1}{n}\left[u_{1}^{\prime}\left(y^{L}\right)-u_{2}^{\prime}\left(y^{L}\right)\right]
$$

which has the same interpretation as (21). To be more precise, (31) can be seen as a special case of (21) when $\lambda$ tends to infinity. Observe that we can think about $\lambda \rightarrow \infty$ as the case where a regulated firm faces an exogenous revenue requirement which equals the (private) monopoly profits. Put differently, we have the standard result that when $\lambda$ becomes arbitrarily large, the pricing policy of a regulated firm coincides with that 
of a private monopoly; see Cremer and Laffont (2003). One readily verifies that this property also applies under Regime $B$, when $y^{H}=y^{L}=y$.

Finally, it is convenient to define $\pi^{m}(e)$ as the profit achieved by the monopoly. It is obtained by substituting the solution to the monopoly problem into (7).

\subsection{Level of enforcement, $e$}

Assume first that $e$ is chosen by the monopoly. The first-order condition is then given by

$$
\frac{d \pi^{*}(e)}{d e}=-1+\eta_{2} k_{e}\left(y^{L P}, e\right)=-1+(n+1) k_{e}\left(y^{L P}, e\right)=0
$$

where we have used the envelope theorem and (30). Rearranging this condition, yields

$$
k_{e}\left(y^{L P}, e\right)=\frac{1}{(n+1)},
$$

which is again a limit case (for $\lambda$ tending to infinity) of the expression (22) obtained for the regulated firm. Observe that the RHS of (33) is smaller than the RHS of (22) (for any finite level of $\lambda$ ). Using (25), it follows that a private monopoly spends more on enforcement than a regulated firm. This result does not come as a surprise. The higher is the level of $\lambda$, the more eager is the firm to extract rents from consumers. Recall that the benefit of an increase in $e$ is associated with its impact on rents through the participation constraint of type 2 individuals.

Now assume that $e$ is set by a welfare maximizing public authority, while the remaining variables continue to be chosen by the profit maximizing monopoly. Social welfare (total surplus) evaluated at the monopoly solution is given by ${ }^{17}$

$$
W=\left[u_{1}\left(y^{L}\right)-p^{L}-K\right]+n\left[\theta u_{2}\left(y^{L P}\right)-k\left(y^{L P}, e\right)\right]+\pi^{m}(e) .
$$

The first term in brackets is the utility of type 1 individuals (with a binding incentive constraint, we have $\left.u_{1}\left(y^{H}\right)-p^{H}=u_{1}\left(y^{L}\right)+p^{L}-K\right)$. The second term is the utility of type 2 individuals (determined by the participation constraint) while the third term represents (monopoly) profits. Observe that $y^{L}$ is determined by (31) and is independent

\footnotetext{
${ }^{17}$ We use unweighted surplus. This means that we implicitly assume that the monopoly can generate enough revenue to cover its cost but that profits cannot be taxed away.
} 
of $e$. However, $p^{L}$ does depend on $e$. To make the expression more tractable we can eliminate this variable by using the participation constraint of type 2 which yields

$$
W=u_{1}\left(y^{L}\right)-u_{2}\left(y^{L}\right)-K+(n+1)\left[\theta u_{2}\left(y^{L P}\right)-k\left(y^{L P}, e\right)\right]+\pi^{m}(e) .
$$

With $y^{L}$ independent of $e$ and $y^{L P}$ determined by (3) we can use the envelope theorem to differentiate $W$ yielding

$$
\begin{aligned}
\frac{\partial W}{\partial e} & =-(n+1)\left[k_{e}\left(y_{2}^{p}, e\right)\right]+\frac{d \pi^{m}(e)}{d e} \\
& =-(n+1)\left[k_{e}\left(y_{2}^{p}, e\right)\right]+\left[(n+1)\left[k_{e}\left(y_{2}^{p}, e\right)\right]-1\right]=-1 .
\end{aligned}
$$

Consequently, the optimal level of e is equal to zero. In other words, when the information good is provided by a (profit maximizing) monopoly public authorities should not spend money to combat piracy. The intuition is very simple: in our setting, expenditures on $e$ have no impact on $y^{L}$ and $y^{H} .{ }^{18}$ Their only impact is to reduce the reservation utility of type 2 individuals and thus to increase monopoly profits. This appears in (34) where the increase in profits (second term) is exactly cancelled out by the reduction in consumer surplus. To sum up, the threat of piracy limits monopoly rents. There is no reason for public authorities to spend money on enforcement.

So far we have concentrated on Case $I$, where the firm sells the good to the two types of consumer. However, it is clear that Case $I I$ is also possible and, as a matter of fact, will occur "more often" (for a larger set of parameter values) with a private monopoly than with a regulated firm. Now, in Case $I I, e$ is set at zero, whatever the objective of the authority which controls it. Consequently, the result that the (socially) optimal level of enforcement under monopoly provision is zero continues to hold in Case $I I$. Nevertheless there is one important caveat to this result. To derive it we have implicitly assumed that the case chosen by the firm does not depend on the level of $e$. Now, one can easily verify that this may or may not be true depending on the specificities of the setting. The problem is that the higher is $e$, the more attractive is Case $I$ to the firm. Consequently, it is not impossible that Case $I$ emerges for high levels of $e$, while Case $I I$ is relevant for low levels of $e$. In this case the determination of the optimal level is a

\footnotetext{
${ }^{18}$ This is dues to the quasi-linearity of preferences.
} 
more complicated exercise. Specifically, it may then be optimal to set a level of $e$ that is positive and just sufficient for the monopoly to choose Case $I$.

\section{Conclusion}

When dealing with information goods such as DVD, CD or software the issue of illegal copying cannot be avoided. Firms, regulated or not, can use both prices and piracy control to obtain the maximum consumer surplus or profit respectively. We show in this paper that allowing for piracy may be desirable not only for the maximization of profit but also for that of social welfare. We distinguish among three settings: regulated monopoly, unregulated private monopoly and a private monopoly with piracy control enforced by a social planner. We show that in a world of asymmetric information piracy control can be used to relax some of the incentive compatibility constraints, particularly that preventing customers of the primary market from shifting to the secondary market. Furthermore, three levels of piracy control emerge. The highest is the one chosen by the private monopoly when there is no interference from a regulator. The next level is the one chosen by the regulated monopoly. The lowest, that can be zero, is the level of control chosen by the public authority in case of private monopoly.

Among some of the limitations of our approach there is the social objective that assumes away redistributive considerations. Piracy can be desirable not only for its effect on self-selection constraints, but also for its redistributive implications. This question is clearly on our research agenda. Similarly, one may want to explore the relationship between piracy and the degree of risk aversion. This is done in a different, but related context, namely insurance fraud, by Pestieau et al. (2003) who show that it can pay for the insurance sector to let some insurees fraud when they have a quite low risk aversion, their number is small and the cost of control is very high. 


\section{References}

[1] Belleflamme, P., (2003), Pricing information goods in the presence of copying, in: Gordon, W.J. and R. Watt, eds., The Economics of Copyright: Developments in Research and Analysis, Edward Elgar, UK

[2] Boe, S. SH. and J-P. Choi, (2003), A model of piracy, unpublished.

[3] Comer, K. and R. Rumelt, (1991), Software piracy: a strategic analysis of protection, Management Science, 37, 125-139.

[4] Peitz, M. and P. Waelbroeck, (2003), Piracy of digital products: a critical review of the economics literature, CESifo WP 1122.

[5] Pestieau, P., U. Possen and S. Slutsky, (2003), Optimal insurance pricing when individuals have different risk aversion, unpublished.

[6] Romer, P., (2002), When should we use intellectual property rights?, American Economic Review, 92, 213-216.

[7] Shy, O. and J.-F. Thisse, (1999), A strategic approach to software protection, Journal of Economics Management Strategy, 8, 163-190.

[8] Takeyama, L., (1994), The welfare implications of unauthorized reproduction of intellectual property in the presence of network externalities, Journal of Industrial Economics, 42, 155-166.

[9] Yoon, K., (2002), The optimal level of copyright protection, Information Economics and Policy, 14, 327-348. 